\title{
El rol de las organizaciones de usuarios de agua en el aprovechamiento sostenible de aguas subterráneas en Perú( ${ }^{*}\left({ }^{* *}\right)$
}

\section{The role of water user organizations in the sustainable use of groundwater in Peru}

\author{
Paul Villegas Vega ${ }^{(* * *)}$ \\ Pontificia Universidad Católica del Perú (Lima, Perú)
}

\begin{abstract}
Resumen: En el presente artículo, el autor analiza la manera en la que las organizaciones de usuarios de agua contribuyen al aprovechamiento sostenible de las aguas subterráneas en el Perú. Para ello, comienza describiendo los instrumentos normativos provistos en el país para la gestión del recurso hídrico en mención y el régimen aplicable para su aprovechamiento por parte de los usuarios de agua, a partir de los cuales se conforman las organizaciones de usuarios de agua; seguido por la presentación de la figura de dichas organizaciones como actores que coadyuvan a la sostenibilidad de las aguas subterráneas y; finaliza con un breve análisis sobre la sostenibilidad de las aguas subterráneas a partir del aprovechamiento por parte de las organizaciones de usuarios de agua.
\end{abstract}

Palabras clave: Derecho de aguas - Organizaciones de usuarios del agua - Aguas subterráneas - Sostenibilidad

\begin{abstract}
In this article, the author analyzes how water user organizations contribute to the sustainable use of groundwater in Peru. In order to do that, he begins by describing the regulatory instruments provided in the country for water resource management and the applicable regime for its use by water users, from which water users' organizations are formed; after that, he presents the figure of these organizations as actors who contribute to the sustainability of groundwater and; finally, he makes a brief analysis of the sustainability of groundwater from the use by water users' organizations.
\end{abstract}

Key words: Water law - Water user organizations - Groundwater - Sustainability

$\left(^{*}\right) \quad$ Nota del Editor: Este artículo fue recibido el 30 de agosto de 2020 y su publicación fue aprobada el 8 de octubre de 2020.

$\left({ }^{* *}\right) \quad$ El presente trabajo ha sido realizado en el marco del proyecto de investigación "Protección de las Aguas Subterráneas. Análisis sobre la sostenibilidad de su aprovechamiento y su vinculación con el Derecho Humano al Agua y Saneamiento", financiado por el Vicerrectorado de Investigación de la Pontificia Universidad Católica del Perú PUCP. Se agradece el apoyo de Marycielo Miranda Cabrera y Braylyn Paredes Aranda, egresada y estudiante de la Facultad de Derecho de la PUCP, respectivamente, en la elaboración del presente artículo.

$\left.{ }^{(* * *}\right)$ Profesor de Derecho Administrativo de la Pontificia Universidad Católica del Perú PUCP e investigador en el Grupo de Investigación en Derecho Administrativo GIDA de la misma casa de estudios. ORCID: https://orcid.org/0000-0003-2511-7240. Correo electrónico: villegas.paul@pucp.edu.pe 


\section{Introducción}

El agua subterránea es un recurso vital para el bienestar del ser humano al tener un rol fundamental en el suministro seguro y asequible del agua potable en el ámbito urbano y rural. Pese a su importancia, históricamente, el mencionado recurso ha sido expuesto a situaciones de riesgo (Foster et al., 2002, p. 2; Villar, 2016, pp. 93-96) generadas por la urbanización, el desarrollo industrial, las actividades agrícolas, emprendimientos mineros, grandes proyectos de infraestructura, entre otros.

De ahí que resulte necesario que los diferentes países, incluido Perú, generen mecanismos de protección para asegurar la sostenibilidad del recurso hídrico, aun cuando sea aprovechado en las distintas actividades que emprende o realiza el hombre. En el mismo sentido, Foster et al. (2002, p. 2) señalan que las acciones de los Estados deberán basarse en criterios de sustentabilidad ambiental, reconociéndose así la relevancia de proteger elementos clave (como las aguas subterráneas) para mantener el equilibrio de los ecosistemas.

Entre las alternativas de acción para lograr la sostenibilidad de las aguas subterráneas y, por ende, contrarrestar los problemas de la sobreexplotación o la contaminación del recurso hídrico, López-Vera (2012, p. 149) destaca la implementación de reformas legales y la formación de comunidades de usuarios de agua subterránea. Así, en países como Perú se han implementado instrumentos normativos con el objeto de gestionar adecuadamente el recurso y regular la actividad de los actores vinculados a la misma. Entre estos últimos se encuentran las organizaciones de usuarios de agua, las cuales son creadas para alcanzar la sostenibilidad de las aguas subterráneas en el marco de su aprovechamiento.

Con el propósito de analizar la manera en que las organizaciones de usuarios de agua contribuyen a la sostenibilidad de las aguas subterráneas en Perú, se ha dividido el presente trabajo en tres secciones: en la primera, se tratan los instrumentos normativos aplicables a la gestión de los recursos hídricos, incluidas las aguas subterráneas, así como el régimen jurídico aplicable para el aprovechamiento de las aguas subterráneas en el país; en la segunda, se describen las organizaciones de usuarios de agua como actores y como colaboradores en la sostenibilidad de los recursos hídricos como las aguas subterráneas, desde su participación en la gestión integral de recursos hídricos; $y$, en la tercera, se analiza la contribución de estos últimos actores para la sostenibilidad de las aguas subterráneas, para así evitar situaciones de sobreexplotación de dichos recursos hídricos.

\section{Instrumentos normativos en la gestión de las aguas subterráneas y régimen jurídico para su aprovechamiento}

Para la gestión de las aguas subterráneas, se debe tener en consideración los instrumentos normativos que brindan un marco de actuación en el sector hídrico, así como el régimen jurídico aplicable para el uso de las aguas subterráneas.

\subsection{Instrumentos normativos para la gestión de las aguas subterráneas}

Así, sobre los instrumentos normativos (o legales) que sean creados con propósito de una gestión adecuada de los recursos hídricos, se debe señalar que estos deberán ser específicos, en tanto versarán sobre las aguas subterráneas como es en el presente caso. El deber de formulación de instrumentos normativos que permitan una adecuada gestión de recursos hídricos se base en la obligación del Estado peruano de "garantizar el acceso al recurso y el manejo sostenible de este", según refiere el Tribunal Constitucional (fundamento 19, 2020).

En el país, estos instrumentos se consolidan en el Sistema Nacional de Gestión de los Recursos Hídricos (SNGRH), el cual fue creado para articular el accionar del Estado en los procesos de gestión integrada y conservación de los distintos recursos hídricos -dentro de ellos, el agua subterránea-, y para crear espacios de coordinación y participación de los diferentes agentes involucrados que establece la Ley 29338 - Ley de Recursos Hídricos.

Esta norma establece que los objetivos del SNGRH, cuyo ente rector es la Autoridad Nacional del Agua (ANA), son los siguientes (artículo 12 de la Ley de Recursos Hídricos):

a) Coordinar y asegurar la gestión integrada y multisectorial, el aprovechamiento sostenible, la conservación, el uso eficiente y el incremento de los recursos hídricos, con estándares de calidad en función al uso respectivo; y, 
b) Promover la elaboración de estudios y la ejecución de proyectos y programas de investigación y capacitación en materia de gestión de recursos hídricos.

En materia de aguas subterráneas, los objetivos del SNGRH impactan de manera especial en la exploración y explotación de estos recursos. Y es que la Administración Pública detenta y desarrolla la planificación de la explotación sostenible, el otorgamiento de los títulos habilitantes, la supervisión de la actuación de los actores conforme los estándares establecidos, entre otros, a partir de los cuales debe asegurar la sostenibilidad de dichos recursos.

De lo mencionado sobre la planificación, se debe señalar que esta debe ser entendida como la identificación de objetivos y el establecimiento (o elección) de medios para la consecución de aquellos objetivos, mediante un proceso de racionalización (Lozano, 2015, p. 165). El Título VII de la Ley de Recursos Hídricos menciona que la planificación de la gestión de recursos hídricos comprende los siguientes instrumentos (artículo 44):

1. La Política Nacional Ambiental;

2. La Política y Estrategia Nacional de Recursos Hídricos;

3. El Plan Nacional de los Recursos Hídricos; y

4. Los Planes de Gestión de Recursos Hídricos en las Cuencas.

Ahora bien, estos instrumentos de planificación de los recursos hídricos no son una mera manifestación del reconocimiento del deber de garantizar el acceso al recurso hídrico y manejo sostenible del mismo como ya se ha mencionado, sino que, además, implica que el Estado deba efectivizar dicho deber de garantía a través de sus actuaciones, a fin asegurar la sostenibilidad del recurso hídrico.

En particular, la vinculación de los instrumentos referidos a las aguas subterráneas, se evidencia en la explotación de dichos recursos, en la cual se debe considerar la disponibilidad de los recursos superficiales y subterráneos de manera conjunta. De ahí que, conforme anotan Cairampoma y Villegas, sea indispensable que se conozca la cantidad y calidad de los recursos hídricos superficiales y subterráneos a nivel de cuenca (2016, p. 153), sobre los cuales se otorgarán derechos de uso.

Con el propósito de conocer el objetivo de cada uno de los instrumentos en razón de la gestión de recursos hídricos y, con ello, las aguas subterráneas, mencionaremos brevemente los instrumentos de planificación y su relación (o impacto) en los recursos hídricos subterráneos.

En primer lugar, sobre la Política Nacional Ambiental, aprobada por el Decreto Supremo 012-2009-MINAM, debemos señalar que establece los lineamientos y ejes de política a ser adoptados por los diferentes niveles de gobierno (nacional, regional y local) para la mejora de la calidad de vida de la sociedad y la sostenibilidad de los recursos naturales e, inclusive, los hídricos como las aguas subterráneas.

En segundo lugar, sobre la Política y Estrategia Nacional de Recursos Hídricos, aprobada mediante Decreto Supremo 006-2015-MINAGRI, podemos mencionar que es un instrumento con cinco (05) ejes de acción y objetivos específicos que sirven de guía para las actuaciones de las entidades públicas y agentes privados involucrados en la gestión de los recursos hídricos. De manera que la eficiencia, protección, seguridad, universalidad y promoción de la cultura de los recursos hídricos se encuentran presentes en la gestión y aprovechamiento de las aguas subterráneas al ser un recurso hídrico, conforme se puede desprender en el siguiente cuadro:

\section{Ejes de acción y objetivos de la Política y Estrategia} Nacional de Recursos Hídricos

\begin{tabular}{|c|c|c|}
\hline $\mathrm{N}^{\circ}$ & Ejes de acción & Objetivos \\
\hline 1 & $\begin{array}{l}\text { Gestión de la } \\
\text { cantidad }\end{array}$ & $\begin{array}{l}\text { Conservar los ecosistemas y los } \\
\text { procesos hidrológicos de los que } \\
\text { depende la oferta de los recursos } \\
\text { hídricos para el país y promover } \\
\text { el uso eficiente del agua. }\end{array}$ \\
\hline 2 & $\begin{array}{l}\text { Gestión de la } \\
\text { calidad }\end{array}$ & $\begin{array}{l}\text { Promover la protección y recu- } \\
\text { peración de la calidad de los } \\
\text { recursos hídricos. }\end{array}$ \\
\hline 3 & $\begin{array}{l}\text { Gestión de la } \\
\text { oportunidad }\end{array}$ & $\begin{array}{l}\text { Atender de manera oportuna la } \\
\text { demanda de los recursos hídri- } \\
\text { cos, respetando el principio de } \\
\text { seguridad jurídica, mejorando su } \\
\text { distribución inclusiva, temporal y } \\
\text { espacial; promoviendo el acceso } \\
\text { universal al agua potable. }\end{array}$ \\
\hline 4 & $\begin{array}{l}\text { Gestión de la cul- } \\
\text { tura del agua }\end{array}$ & $\begin{array}{l}\text { Promover una cultura del agua } \\
\text { para la gestión eficiente y valo- } \\
\text { ración de los recursos hídricos. }\end{array}$ \\
\hline 5 & $\begin{array}{l}\text { Adaptación al } \\
\text { cambio climático } \\
\text { y eventos extre- } \\
\text { mos }\end{array}$ & $\begin{array}{l}\text { Reducir la vulnerabilidad de la po- } \\
\text { blación, aplicando el enfoque de } \\
\text { la Gestión Integrada de Recursos } \\
\text { Hídricos y la Gestión de Riesgos. }\end{array}$ \\
\hline
\end{tabular}

Elaboración Propia.

Fuente: Política y Estrategia Nacional de Recursos Hídricos, 2015.

En tercer lugar, sobre el Plan Nacional de Recursos Hídricos, aprobado mediante Decreto Supremo 013-2015-MINAGRI, se establece las acciones, costos y las 
diferentes fuentes de financiamiento necesarias para alcanzar o materializar los objetivos establecidos de la Política y Estrategia Nacional de Recursos Hídricos. Es así que, según reconoce la misma Autoridad Nacional del Agua sobre el Plan Nacional de Recursos Hídricos, el país debe realizar inversiones que permitan lograr los objetivos establecidos (2016, p. 27):

El Plan establece que al año 2021, nuestro país debe invertir al menos 85196 millones de soles en acciones estructurales (obras físicas) y no estructurales (captación, cultura del agua, desarrollo normativo, etc.); y en el periodo que va del año 2022 al 2035 deberíamos invertir 60381 millones de soles, lo que hace un total de 145578 millones de soles para atender las demandas de aguas.

En atención a la consecución de los objetivos establecidos en el Plan Nacional de Recursos Hídricos, se desarrollaron once (11) estrategias de intervención que, a su vez, están compuestos por treinta (30) programas sectoriales especíicos, los cuales tienen por finalidad atender las diferentes demandas de agua por parte de la población. En estos programas, se pueden observar tres (03) estrategias de intervención que están referidas a las aguas subterráneas, siendo estas las siguientes:

a) La primera estrategia de intervención hace referencia a la mejora del conocimiento de los recursos y las demandas. En ella, se detalla como programa sectorial el aumento del conocimiento de las aguas subterráneas, para lo cual se designa una inversión aproximada de 128 millones de soles al 2021.

b) La segunda estrategia contiene un programa especial para el recurso hídrico subterráneo, el cual está orientado al aumento de la disponibilidad en cuanto a la gestión de acuíferos sobreexplotados, para el cual se dispone que se inviertan un aproximado de 52 millones de soles al 2021.

c) La tercera estrategia se basa en la mejora del conocimiento de la calidad de las aguas. En este, se ha incluido un programa específico para la calidad de las aguas subterráneas que considera una inversión aproximada de 40 millones de soles al 2021.

Adicionalmente a las estrategias expuestas, el Plan Nacional de Recursos Hídricos considera otras tres (03) estrategias que aportan al perfil de gestión integrada de recursos hídricos en Perú. La primera se centra en la implementación de la gestión integrada de los recursos hídricos y cuenta con (02) dos programas sectoriales direccionados al fortalecimiento institucional y administrativo de la Gestión Integrada de Recursos Hídricos. La segunda se refiere a la coordinación institucional y gobernanza hídrica en la que se encuentra el programa de participación y consulta. Y la tercera se concentra en la intervención destinada a la educación ambiental y cultura del agua, la cual incluye programas de gestión del conocimiento y cultura del agua, y programas sobre comunicación, sensibilización y concienciación de la gestión integrada.
Es a partir de la sumatoria de las estrategias mencionadas, que se puede realizar una gestión unificada de los recursos hídricos, tomando en consideración la realidad de cada cuenca hidrográfica y sumando a actores del gobierno nacional, regional y local. En este punto resultará indispensable el rol protagónico de la Autoridad Nacional del Agua (ANA, 2016, p. 28; Parejo, 2012, pp. 187-193) para articular los distintos niveles de gobierno acorde con el rol rector que detenta en el Sistema Nacional de Gestión de los Recursos Hídricos.

En cuarto y último lugar, sobre los Planes de Gestión de Recursos Hídricos de las Cuencas, generados a partir de los "Lineamientos para la formulación de los Planes de Gestión de Recursos Hídricos en las Cuencas", aprobado por Resolución Directoral 006-2015-ANA-DCPRH, debemos señalar que son instrumentos públicos vinculantes, de actualización periódica y revisión justificada. Por lo tanto, no generan derechos en favor de particulares o entidades públicas y su modificación; es decir, que no puede afectar derechos previamente otorgados y no da lugar a indemnización.

Estos Planes de Gestión de Recursos Hídricos de las Cuencas permiten la planificación y participación de todos los actores involucrados en relación a una cuenca en específico, siendo uno de ellos las organizaciones de usuarios de agua como las juntas de usuarios de agua. Según la Autoridad Nacional del Agua, el Banco Interamericano de Desarrollo y el Banco Mundial (sf, p. 3), esta planificación o Gestión Integrada de los Recursos Hídricos conlleva un enfoque multisectorial, dividido en tres enfoques, según se observa a continuación:

a) Esfera social: Necesidades y deseos, qué modelo de cuenca se quiere, cuál es la capacidad de pago, cuáles son las prioridades, a qué se está dispuesto a renunciar y a qué no, etcétera.

b) Esfera ambiental: Qué recursos están disponibles, en cuáles hay crisis, qué zonas se deben preservar, cómo hacer sostenible ambientalmente la explotación del recurso, en qué condiciones se les quiere dejar a las generaciones futuras, etc. 
c) Esfera económica: Qué modelo económico y territorial se diseña en el país, qué actividades necesitan agua como insumo, en qué cantidad, con qué disponibilidad tecnológica, qué modelo de sostenibilidad económica se puede plantear (se debe pensar en explotación y mantenimiento, no solo en inversión).

Este enfoque multisectorial descrito, se refleja en tres prácticas que permitirán que todos los actores de la cuenca involucrados en la gestión del recurso hídrico reflexionen y determinen estrategias de cambio a futuro: (i) planificación de los recursos hídricos como un elemento esencial para una adecuada gestión de los recursos, (ii) la participación organizada de los usuarios y (iii) el modelo colaborativo para la proyección de los diferentes escenarios en la gestión de recursos hídricos.

En función a lo expuesto en este apartado, podemos señalar que, en Perú, se cuenta con cuatro instrumentos que permiten materializar la planificación de la gestión de los recursos hídricos, atendiendo a la especialidad que reportan los recursos hídricos subterráneos, los cuales, al igual que los superficiales, presentan características propias en cada cuenca hidrográfica.

\subsection{Régimen aplicable para el aprovechamiento de las aguas subterráneas}

Por su parte, sobre el régimen aplicable para el aprovechamiento de las aguas subterráneas, debemos partir por reconocer que la gestión adecuada de los recursos hídricos en Perú tiene como punto de partida los instrumentos descritos en el apartado anterior, en tanto que se constituyen como la herramienta básica en la regulación para el aprovechamiento de los recursos hídricos, tanto superficiales como subterráneos (Lozano, 2015, p. 166).

En concordancia con lo señalado, se establece un sistema de derechos de agua que brinda beneficios, en tanto permite la posibilidad de explotar y conservar los recursos hídricos (Garduño et al., 2006, p. 1); es decir, colabora con un escenario de aprovechamiento sostenible.

Sobre el particular, la Ley de Recursos Hídricos ha establecido que el reconocimiento de derechos de agua es a través de títulos habilitantes, los denominados derechos de uso en el ordenamiento jurídico peruano. Estos derechos de uso permiten a quien los detenta aprovechar los recursos hídricos conforme una adecuada gestión de los mismos, debiendo ser coherente con la cohesión social (Espinosa-Saldaña, 2020). En materia de aguas subterráneas, los títulos habilitantes se refieren a los derechos de uso para la exploración o explotación del recurso hídrico en mención.

En el artículo 110 de la Ley de Recursos Hídricos, se señala que los derechos de uso para aguas subterráneas se regularán por lo dispuesto en su Título IV. Adicionalmente, aquel artículo señala que, en el caso de cese temporal o permanente del uso, los titulares de estos derechos están obligados, bajo responsabilidad, a tomar las medidas de seguridad necesarias que eviten daños a terceros; $y$, que los usuarios de aguas subterráneas deberán instalar y mantener piezómetros en cantidad y separación determinados por la autoridad respectiva, donde registren la variación mensual de los niveles freáticos, información que deben comunicar a la Autoridad Nacional del Agua.

En el Reglamento de la Ley de Recursos Hídricos, se especifican las condiciones necesarias para otorgar derechos de uso en materia de aguas subterráneas, conforme se exponen a continuación (artículo 241):

a) Que su ejercicio no interfiera o altere el ejercicio de otros derechos de uso de agua superficial o subterránea otorgados con anterioridad.

b) Que la extracción de agua subterránea no cause fenómenos físicos, químicos o ambos, que alteren perjudicialmente las condiciones del reservorio acuífero, las aguas allí contenidas, ni el área superficial comprendida en el radio de influencia del pozo cuando abarque terrenos de terceros.

c) Que no produzcan interferencia con otros pozos u otras fuentes naturales de agua.

d) Que exista la disponibilidad del agua subterránea solicitada y que sea apropiada en calidad, cantidad y oportunidad para el uso al que se destinará.

e) Que las obras hidráulicas de alumbramiento o recarga artificial del acuífero, conducción, utilización, medición y las demás que fuesen necesarias cuenten con la aprobación de la Autoridad Nacional del Agua.

Además de los requisitos establecidos para el otorgamiento de derechos de uso de agua subterránea, se requiere el cumplimiento de lo establecido en el artículo 53 de la Ley de Recursos Hídricos, a través del cual se establecen condiciones que buscan asegurar su aprovechamiento en condiciones de sostenibilidad. Estas condiciones son las siguientes:

a) Que exista la disponibilidad del agua solicitada y que ésta sea apropiada en calidad, cantidad y oportunidad para el uso al que se destine; 
b) Que la fuente de agua a la que se contrae la solicitud tenga un volumen de agua disponible que asegure los caudales ecológicos, los niveles mínimos de reservas o seguridad de almacenamiento y las condiciones de navegabilidad, cuando corresponda y según el régimen hidrológico;

c) Que no ponga en riesgo la salud pública y el ambiente;

d) Que no se afecte derechos de terceros;

e) Que guarde relación con el plan de gestión del agua de la cuenca;

f) Que el interesado presente el instrumento ambiental pertinente aprobado por la autoridad ambiental sectorial competente; $y$

g) Que hayan sido aprobadas las servidumbres, así como las obras de captación, alumbramiento, producción o regeneración, conducción, utilización, avenamiento, medición y las demás que fuesen necesarias.

De lo mencionado en las normas citadas, tenemos que el aprovechamiento de las aguas subterráneas es a través de los títulos habilitantes que reconocen derechos de uso, conforme señalan Cairampoma y Villegas (2016, p. 155). No obstante, en atención al deber del Estado de garantizar el acceso al recurso hídrico y su sostenibilidad, en el ordenamiento jurídico se ha establecido condiciones a cumplir para el otorgamiento de dichos títulos habilitantes. De manera que los próximos a ser usuarios de agua deben cumplir con dichas condiciones a efectos aprovechar el recurso hídrico de aguas subterráneas.

En ese sentido, a partir de lo expresado en esta sección, podemos señalar que el Estado peruano tiene el deber de garantizar el acceso a los recursos hídricos como es el caso de las aguas subterráneas y asegurar su sostenibilidad, para lo cual ha provisto de instrumentos normativos que establecen los lineamientos para el manejo y gestión de recursos hídricos como son las aguas subterráneas, así como un régimen jurídico que establece condiciones de sostenibilidad para el otorgamiento de derechos de uso de dichos recursos hídricos.

\section{Organizaciones de Usuarios de Agua}

El aprovechamiento de las aguas subterráneas ha ido incrementando con el tiempo de una manera desorganizada (Muñoz, 2016; Pino, 2019), situación que ha generado un gran desafío para los encargados de su administración, como es el caso de las organizaciones de usuarios de agua.

Los usuarios de aguas subterráneas, al ser los solicitantes o tenedores de un derecho de uso de agua, son los actores más importantes en la gestión de un sistema de derechos de agua subterránea, conforme señala Garduño et al. (2006, p. 4). Estos actores tienen intereses directos-conforme, en su momento, García de Enterría (1984) expuso de manera general al desarrollar el concepto de pouvoir municipal- en las aguas subterráneas, y se relacionan con otros actores o posibles actores involucrados en la gestión del mencionado recurso, sean del sector público, sector privado e, inclusive, la sociedad misma. Esto supone que se genere una relación de cooperación en el desarrollo de la actividad, aunque a la fecha, según reconoce Groundwater Governance (2016, p. 12), en la mayoría de los países todavía no existe una cooperación efectiva entre los otros actores mencionados y los propios usuarios.

Ahora bien, estos usuarios agrupados en organizaciones de usuarios de agua han tenido un rol importante en la gestión del uso sostenible de los recursos hídricos, al ser integrantes del Sistema Nacional de Gestión de Recursos Hídricos (artículos 10 y 16 de la Ley de Recursos Hídricos) y, en particular, de las aguas subterráneas, al tener como finalidad la participación organizada de los usuarios en dicha actividad.

Así, con el propósito de analizar el impacto de estas organizaciones en el aprovechamiento de las aguas subterráneas, resulta pertinente presentarlas de manera que el lector pueda conocer su naturaleza e identificarlas en su campo de actuación en relación a este recurso hídrico en específico. A continuación, se desarrollan primero sus aspectos generales para después analizar los niveles de organización que ellas mantienen.

\subsection{Aspectos generales}

Los usuarios de agua, conforme el artículo 39 del Reglamento de la Ley de Recursos Hídricos, son titulares de derechos de uso de agua para el abastecimiento del recurso a través de los servicios que brindan las organizaciones de usuarios de agua, bien a través de juntas, comisiones o comités de usuarios de agua. Estos usuarios pueden formar parte de las organizaciones de usuarios de agua, en tanto que comparten una fuente superficial o subterránea y un sistema hidráulico común, conforme al artículo 39 del cuerpo normativo previamente citado.

La Ley de Recursos Hídricos precisa, en su artículo 27 , que los usuarios pueden 
organizarse en asociaciones civiles para efectivizar su participación en la gestión multisectorial y de uso sostenible de los recursos hídricos; para lo cual el Estado garantiza su autonomía y elección democrática a través del reconocimiento por parte de la Autoridad Nacional de Agua. Cabe señalar, en el mismo sentido de lo expresado por el Tribunal Constitucional (2020) en el fundamento 44, que estas asociaciones cuentan con un régimen distinto a las reguladas por el Código Civil por las actividades que desarrollan.

Estas organizaciones de usuarios del agua son definidas como organizaciones sin fines de lucro conformadas por personas naturales y jurídicas, quienes poseen derecho de uso de agua otorgado por la autoridad competente (artículo 3 del Reglamento de la Ley de Organizaciones de Usuarios de Agua, Decreto Supremo No 0005-2015-MINAGRI) y canalizan la participación de sus miembros en la gestión multisectorial y de uso sostenible de los recursos hídricos (artículo 2 de la Ley de las Organizaciones de Usuarios de Agua), en tanto son reconocidos como integrantes del Sistema Nacional de Gestión de Recursos Hídricos (artículo 11 de la Ley de Recursos Hídricos).

Al respecto, el Tribunal Constitucional (2020), en su fundamento 40 , señala que las organizaciones de usuarios de agua (i) gestionan recursos de titularidad del Estado y (ii) desarrollan funciones administrativas en torno a dichos recursos. Por tanto, las referidas organizaciones, finalmente particulares, se encuentran desarrollando tareas de interés general, colaborando así con los fines del Estado.

\subsubsection{Constitución y personería jurídica de las organizaciones de usuarios de agua}

La Ley de las Organizaciones de Usuarios de Agua (artículo 4) y su Reglamento (artículos 16 y 17) establecen que las juntas y comisiones se constituyen sobre la base de sectores o subsectores hidráulicos, los cuales deben estar delimitados previamente por la Autoridad Nacional del Agua. Y prohíbe que pueda constituirse dos o más organizaciones del mismo nivel en el mismo sector o subsector.

Los usuarios de agua que cuentan con sistemas de abastecimiento propio podrán constituir organizaciones de usuarios de agua de carácter regional o nacional, a fin de canalizar su participación en la gestión multisectorial de los recursos hídricos y de defender sus derechos e intereses comunes.

Para su reconocimiento, conforme el artículo 23 del Reglamento de la Ley de Organizaciones de Usuarios de Agua, deberán cumplir con los siguientes requisitos: acta de constitución (que considere el acuerdo de constituir organización a nivel regional o nacional, constituida exclusivamente por usuarios con sistema de abastecimiento propio, la denominación, la identificación de sus integrantes y de su directiva), patrimonio y propuesta de estatuto.
Una vez constituidas, las organizaciones de usuarios de agua adquieren personería jurídica con su inscripción en los registros públicos. Para tal inscripción, resulta de carácter obligatorio que, previamente, cuenten con resolución administrativa por parte de la Autoridad Nacional del Agua que señale que sus domicilios se encuentran registrados dentro del ámbito territorial en el que se organizan.

Cabe mencionar que la inscripción es obligatoria para la junta de usuarios y facultativa para las comisiones y comités de usuarios, vinculando dicha calificación registral al reconocimiento otorgado por la Autoridad Nacional del Agua a través de la resolución administrativa.

Además, una vez inscrita la organización de usuarios de agua, no será necesario que se inscriba cada acto o decisión adoptada como las elecciones de representantes orgánicos, voluntarios, adecuaciones, modificaciones estatutarias, entre otros; debido a que se podrá solicitar instrumentos complementarios que le permitan el control de legalidad y adecuación de los mismos (SUNARP, 2018, p. 32).

Las restricciones que en esta sección se mencionan ponen en evidencia, en el mismo sentido del fundamento 54 del Tribunal Constitucional (2020), que las organizaciones de usuarios de agua si bien son consideradas como asociaciones por su constitución fruto de la voluntad de los usuarios, es en base a la relevancia de su actuación (prestación de un servicio público vinculado a un recurso natural) que se les establece ciertos límites.

\subsubsection{Reconocimiento administrativo de las organizaciones de usuarios de agua} La infraestructura hidráulica menor de carácter público, entendida como estructura empleada para realizar actividades de captación, regulación, distribución, medición y drenaje secundario (artículo 13.2 del Reglamento de Operadores de Infraestructura Hidráulica, Resolución Jefatural 327-2018-ANA), resulta indispensable para el aprovechamiento de los recursos hídricos y tiene como su titular a la Autoridad Nacional del Agua.

El Estado delega el ejercicio de dicha titularidad a las organizaciones de usuarios de agua, mediante el reconocimiento 
administrativo (artículo 20 del Reglamento de la Ley de las Organizaciones de Usuarios de Agua) que otorga la Autoridad Nacional del Agua en una resolución administrativa. Y es que la colaboración de particulares en las actividades propias del Estado resulta parte de la evolución que ha sufrido este último para asegurar sus fines (Parejo, 2012, pp. 35-38), en definitiva vinculados a la dignidad del ser humano.

El reconocimiento en mención implica que, previamente, se haya verificado que la organización de usuarios de agua se encuentre en capacidad de administrar la infraestructura, para ello se realiza una evaluación técnica de los requisitos que debe cumplir. Así, se garantiza que brinde una gestión eficiente y sostenible de los recursos hídricos.

Las organizaciones de usuarios de agua obtienen con este reconocimiento la autorización del Estado para brindar un servicio público, administrar, operar y mantener la infraestructura pública de un sector hidráulico, cobrar tarifas de agua y efectuar la distribución del recurso.

Cabe precisar que el reconocimiento administrativo en cuestión no tiene carácter provisional, sino indeterminado; con ello se busca la permanencia de la participación de los usuarios de agua. No obstante, ello no resta que, en caso se sancione más de tres veces en un periodo no mayor a doce meses a la organización, por parte de la Autoridad Nacional del Agua, ese reconocimiento sea suspendido por resolución administrativa.

\subsubsection{Derechos y deberes de los usuarios}

En el marco del funcionamiento de estas organizaciones, la normativa ha establecido en los artículos 4 y 5 del Reglamento de la Ley de Organizaciones de Usuarios de Agua los siguientes derechos y obligaciones para los usuarios de agua:

\section{A) Derechos:}

- Recibir la dotación de agua conforme al derecho de uso de agua otorgado, disponibilidad del recurso hídrico y programas de distribución de agua aprobados.

- Solicitar información sobre la gestión de su organización de usuarios de agua.

- Acceder, en condiciones de igualdad, a los beneficios y servicios que brinda la organización de usuarios de agua.

- Presentar reclamos ante su organización de usuarios de agua por los servicios brindados, los que serán resueltos en un plazo no mayor de treinta (30) días hábiles.

- Demás derechos establecidos en su Estatuto, la Ley 29338 - Ley de Recursos Hídricos, su Reglamento y demás normatividad aplicable.

\section{B) Obligaciones:}

- Usar el agua en forma eficiente en el lugar y para la finalidad que le fuera otorgada, sin afectar derechos de terceros.
- Participar o contribuir a la conservación, sostenibilidad, mantenimiento y desarrollo de la cuenca y del acuífero.

- Abonar, en forma oportuna, a las juntas de usuarios las tarifas de agua y las retribuciones económicas que se establezcan.

- Cumplir las disposiciones establecidas en la normatividad vigente.

Conforme se puede observar, la normativa ha previsto, por un lado, derechos tendientes a proteger el derecho de uso de agua otorgado a la organización de usuarios a fin de que puedan ejercer plenamente dicho derecho y, por otro lado, obligaciones como límites al ejercicio del derecho de uso de agua para asegurar la sostenibilidad del recurso hídrico. $\mathrm{Y}$ es que, para alcanzar el equilibrio entre el aspecto social, económico y ambiental, se tendrá que considerar la situación particular de la sociedad en conjunto con la disponibilidad hídrica.

\subsubsection{Actividad de las organizaciones}

Las organizaciones de usuarios de agua tienen como principal actividad la gestión de la infraestructura hidráulica y de los recursos hídricos. Conforme lo señala el artículo 13 del Reglamento de la Ley de Organizaciones de Usuarios de Agua, las organizaciones realizan esta actividad conforme al interés público, razón por la cual su gestión deberá ser coherente con el equilibrio que debe mantenerse en una cuenca hidrográfica.

El servicio brindado por las organizaciones de usuarios de agua presenta particularidades en función a la zona geográfica en la cual partícipe (Martín Mateo, 1991, p. 13), así como por las actividades que se desarrollen. No obstante, con la finalidad de contar con homogeneidad entre sus labores, se puede partir por lo señalado en el artículo 14, 16 y 18 del Reglamento de Operadores de Infraestructura Hidráulica y del artículo 9 del Reglamento de la Ley de Organizaciones de Usuarios de Agua, en los que se reconoce los sectores hidráulicos en los cuales actúan las organizaciones de usuarios de agua:

a) En el sector hidráulico mayor está comprendida la infraestructura hidráulica 
mayor, a través de la cual se realizan actividades de (i) trasvase (que deriva el agua de una unidad hidrográfica a otra antigua), (ii) regulación (que almacena y entrega gradualmente el agua), (iii) medición (que determina volúmenes o causales de agua en un punto determinado), (iv) captación (que deriva el agua de su curso natural artificial a una estructura de derivación), (v) derivación (que conduce aguas desde la captación hasta la entrega de las mismas en la infraestructura hidráulica menor o usuarios que no forman parte de un sector hidráulico menor) y (vi) drenaje principal (que evalúa los excedentes de agua desde los drenes secundarios hacia la fuente natural).

b) El sector hidráulico menor comprende la infraestructura hidráulica menor y el ámbito en el que se presta servicio, en la cual se realizan actividades de (i) captación, que deriva el agua de un sector hidráulico mayor o de un curso natural a los sistemas de distribución; (ii) distribución, que traslada las agua desde la captación hasta aquellos usuarios que utilizan el recurso hídrico en una actividad sectorial determinada; (iii) medición, que determina los volúmenes o caudales de agua en un punto determinado de la infraestructura hidráulica; $y$, (iv) de drenaje secundario, que evacúa los excedentes de agua hacia lo drenajes principales.

c) El sector hidráulico de aguas subterráneas considera la infraestructura hidráulica que permite realizar actividades de (i) extracción, (ii) medición, y, (iii) distribución de aguas subterráneas, así como de, (iv) medición de nivel freático, de los parámetros básicos de la calidad y de la explotación de las aguas subterráneas.

En función a la diversidad de infraestructuras que deben ser administradas por las organizaciones de usuarios de agua es que, cinco años después de contar con un nuevo marco normativo como la Ley de Recursos Hídricos, se opta por incorporar al ordenamiento jurídico nacional la Ley, y posterior Reglamento, referida a las organizaciones de usuarios de agua.

\subsubsection{Tarifa de agua cobrada por la organización}

Conforme se establece en el artículo 90 de la Ley de Recursos Hídricos y el artículo 92 del Reglamento de la Ley de Organizaciones de Usuarios de Agua, los titulares de los derechos de agua (o usuarios de agua) deben realizar pagos por el uso de la infraestructura hidráulica pública. Estos pagos son reconocidos en la normativa peruana como las tarifas de agua, las cuales tienen como propósito contribuir al uso sostenible y eficiente del recurso.

Las organizaciones de usuarios cuentan con recursos económicos provenientes de la tarifa por uso de infraestructura hidráulica, de la prestación de otros servicios, donación, legados, otros aportes de los usuarios del sector hidráulico y, en el caso de las aguas subterránea, la tarifa por los servicios de monitoreo y gestión de agua subterránea en atención a lo dispuesto en el artículo 93 del Reglamento de la Ley de Organizaciones de Usuarios de Agua.

Los recursos antes referidos son destinados exclusivamente a solventar los gastos de operación y mantenimiento de la infraestructura hidráulica, excepto aquellos aportes económicos que sean distintos a las tarifas de agua (artículo 94 del Reglamento de la Ley de Organizaciones de Usuarios de Agua).

Resta destacar que las tarifas de agua no son retribuciones económicas por el aprovechamiento de los recursos hídricos; es decir, no estamos frente a la retribución que se realiza por los derechos de uso de agua, la cual es establecida por la Autoridad Nacional del Agua. Por el contrario, estas tarifas de agua son pagos que realizan los usuarios de agua a las organizaciones de usuarios de agua por el uso de la infraestructura hidráulica pública, constituyendo así recursos públicos (artículo 92 del Reglamento de la Ley de Organizaciones de Usuarios de Agua).

\subsubsection{Control de las organizaciones de agua: supervisión y sanción}

La Autoridad Nacional del Agua realiza las acciones de supervisión o fiscalización, de oficio o a petición de un tercio de miembros del Consejo Directivo de una organización de usuarios, para garantizar el normal desarrollo de las funciones de las organizaciones de usuarios de agua, conforme el artículo 12 de la Ley de Organizaciones de Usuarios de agua y el artículo 96 de su Reglamento.

No obstante, sin perjuicio de lo dispuesto en la normativa antes mencionada, será de aplicación lo referido por los artículos comprendidos entre el 239 al 246 del Texto Único Ordenado de la Ley 27444 - Ley de Procedimiento Administrativo General, respecto a la actividad administrativa de fiscalización.

Cabe señalar que la finalidad de la actividad fiscalizadora de la Administración consiste tanto en la verificación del cumplimiento de la legalidad como en la protección de los intereses públicos en juego. Sin embargo, como advierte Izquierdo (2019, p. 416), en los ámbitos sectoriales se pueden encontrar dos 
escenarios respecto a la atribución de la potestad fiscalizadora a un órgano o ente administrativo y las facultades otorgadas en ese ámbito.

El primer escenario se presenta cuando la normativa sectorial solo se limita a atribuir dicha potestad fiscalizadora a un órgano específico, pero sin detallar las facultades que le corresponde. En ese sentido, se deberá aplicar todas las facultades listadas en el artículo 240 del Texto Único Ordenado. Y, en relación al segundo escenario, bajo el cual el legislador sí incluye una enumeración específica de las facultades fiscalizadoras en la normativa sectorial y en ellas no reconoce alguna de las listadas en el artículo 240, se entiende que el órgano fiscalizador deberá respetarlas al tratarse de una concreción lícita del legislador sectorial.

Asimismo, la Autoridad Nacional del Agua tiene facultad de sancionar a las organizaciones de usuarios de agua ante el incumplimiento de funciones a su cargo, a través del procedimiento administrativo sancionador de acuerdo con el 98 del Reglamento de la Ley de Organizaciones de Usuarios de Agua.

Sobre la potestad sancionadora, el artículo $97 \mathrm{del}$ Reglamento de la Ley de Organizaciones de Usuarios de Agua precisa que los miembros de los consejos directivos responden solidariamente con sus organizaciones de usuarios de agua por infracciones en las que esta última incurra. $Y$ es que, a través de las decisiones que aquellos adoptan, la organización de usuarios de agua puede incurrir en alguna de las infracciones previstas en la norma, tales como "no ejecutar las acciones relacionadas a preservar la integridad, idoneidad o el uso adecuado de la infraestructura hidráulica, según los instrumentos técnicos aprobados por la Autoridad Nacional del Agua", "no elaborar o no presentar a la autoridad para su aprobación, dentro del plazo establecido, los instrumentos técnicos necesarios para ejercer el rol de operadores de infraestructura hidráulica, que sean aprobados por la Autoridad Nacional del Agua o el Ministerio de Agricultura y Riego", entre otras infracciones previamente establecidas con las respectivas sanciones a efectos de desincentivar su actuación.

En particular, las infracciones administrativas en las que podrían incurrir las organizaciones de usuarios de agua vinculadas a las aguas subterráneas, conforme al Reglamento, serían (i) las consideradas como leves al estar vinculadas al Plan Multianual de Inversiones, la cual es considerada leve (artículo 106); y, (ii) las consideradas como graves que están relacionadas con la operación y mantenimiento de la infraestructura hidráulica (artículo 105), al incumplimiento de otros instrumentos técnicos (artículo 107), a las tarifas de agua y aportes (artículo 108), a la recaudación y transferencia de la retribución económica (artículo 109), a la distribución de agua (artículo 110) y a la transparencia de la gestión institucional (artículo 111).
En el marco del procedimiento administrativo sancionador que realiza la Autoridad Nacional del Agua, el órgano instructor puede dictar medidas de carácter temporal a fin de garantizar la eficacia de la resolución final y cautelar así el interés público por medio de la ejecución o suspensión de acciones mientras se lleve a dicho procedimiento (artículo $123 \mathrm{del}$ Reglamento de la Ley de Organizaciones de Usuarios de Agua), siempre que se evalúe previamente el riesgo que implicaría su adopción en la gestión de recursos hídricos del sector o subsector hidráulico. Lo mencionado no resta que la adopción de medidas administrativas también es una herramienta que la administración pública podrá aplicar en caso fuese necesario, en ejercicio del poder de policía.

\subsubsection{Disolución de las organizaciones de usuarios de agua}

La disolución de la organización de usuarios de agua es adoptada por la Asamblea General, de conformidad con lo establecido en su estatuto. Una vez adoptada la disolución de la organización de usuarios de agua, el haber neto de la liquidación deberá ser entregado a la organización que asuma las funciones de aquella, de conformidad con lo establecido en el Código Civil (artículo 41 de la ley de recursos hídricos) y, luego, el acuerdo de disolución deberá ser inscrito en la Superintendencia Nacional de los Registros Públicos - SUNARP (2018, pp. 15-16).

De esa manera, la organización de usuarios de agua logra un proceso de disolución ordenado, que atiende a la voluntad de sus miembros, de conformidad con la normativa aplicable y procurando la continuidad de las obligaciones que brindan.

\subsection{Niveles de organización de los Usuarios de Agua}

En Perú se cuenta con tres niveles de organización de usuarios de aguas, encontrándose los comités de usuarios en la base del sector hidráulico, siguiendo las comisiones de usuarios como nivel intermedio y las juntas de usuarios en el tercer nivel. De esta manera, en Perú se ha aprovechado la potestad de organización (Sánchez Morón, 
2011, pp. 223-267) para brindar una estructura lógica que no replique potestades y que permita coordinar entre los niveles mencionados. Al respecto, la diferenciación entre ellas se debe a la distinción de las labores que cada una realiza, escenario que no vulnera el principio de igualdad, conforme lo señala el Tribunal Constitucional (2020) en los fundamentos 57 al 80.

\section{Figura $N^{\circ}$ 1: Organizaciones de Usuarios de Agua}

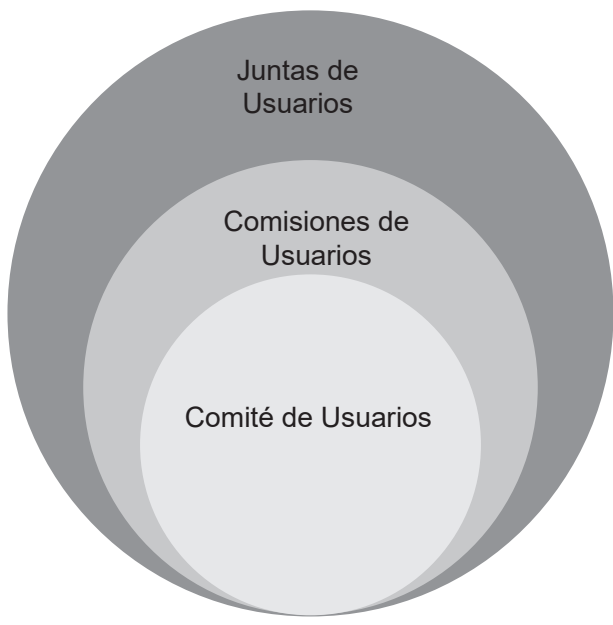

Elaboración propia.

Fuente: Ley de Organizaciones de Usuarios de Agua.

\subsubsection{Comité de usuarios}

El comité de usuarios es la organización de nivel básico, constituida sobre la base de una infraestructura en un subsector hidráulico, que se integra a la comisión de usuarios de agua del subsector hidráulico correspondiente (artículo $62 \mathrm{del}$ Reglamento de la Ley de Organizaciones de Usuarios de Agua).

Entre las funciones del comité de usuarios, la normativa establece a través del artículo 63 del Reglamento las siguientes: (i) canalizar y representar los derechos e intereses de sus integrantes ante la comisión de usuarios a la que se integra; (ii) realizar, por delegación de la comisión de usuarios, las actividades de operación y mantenimiento de la infraestructura hidráulica sobre la cual se organiza; (iii) ejecutar los acuerdos adoptados por la comisión de usuarios a la que integra; (iv) ejecutar las disposiciones que establezca la Autoridad Nacional del Agua; y, (v) promover el uso sostenible y conservación del recurso hídrico.

\subsubsection{Comisión de usuarios}

Las comisiones de usuarios de agua son organizaciones de nivel intermedio, conformadas por usuarios de agua reconocidos en el Registro Administrativo de Derechos de Uso de Agua (RADA), sobre la base un subsector hidráulico (artículo 49 y 54 del Reglamento de la Ley de Organizaciones de Usuarios de Agua).

Previo al reconocimiento, la Autoridad Nacional del Agua solicita opinión a la junta de usuarios a cargo del sector hidráulico al que pertenece el subsector. En caso de no haber junta de usuarios, no será exigible dicha opinión (artículo 22 del Reglamento de la Ley de Organizaciones de Usuarios de Agua).

El Reglamento de la Ley de Organizaciones de Usuarios de Agua, en el artículo 22, establece los requisitos que la comisión de usuarios de agua debe cumplir para obtener el reconocimiento de la Autoridad Nacional del Agua. A continuación, se precisan los requisitos:

- Acta de constitución: acuerdo de usuarios de un mismo subsector hidráulico, denominación, identificación del subsector hidráulico, de los usuarios que conforman la comisión de usuarios y del primer consejo directivo.

- Patrimonio de la comisión.

- Identificación de la infraestructura hidráulica bajo su ámbito territorial.

- Propuesta de estatuto.

- Plano o esquema del sector hidráulico, delimitado por la Autoridad Nacional del Agua.

Entre las funciones de las comisiones de usuarios de agua, conforme al artículo 50 del Reglamento de la Ley de Organizaciones de Usuarios de Agua, se encuentran las siguientes: (i) canalizar y representar los derechos e intereses de los usuarios de agua del subsector hidráulico ante la junta de usuarios; (ii) proponer ante la junta de usuarios el plan de operación, mantenimiento y desarrollo de la infraestructura hidráulica del subsector hidráulico; (iii) realizar, por delegación y condiciones establecidas por parte de la junta de usuarios, las actividades de operación y mantenimiento de la infraestructura hidráulica del subsector hidráulico, distribución del agua en el subsector hidráulico y cobranza de tarifas, 
recaudación de retribución económica y otros aportes económicos; (iv) supervisar las actividades de los comités de usuarios que la integran, en cuanto se refiere a la operación y mantenimiento de la infraestructura hidráulica del subsector hidráulico; (v) ejecutar los acuerdos adoptados por la junta de usuarios que integra así como aquellas actividades que les sean encargadas; (vi) promover el uso sostenible y conservación del recurso hídrico de acuerdo con las disposiciones que establezca la Autoridad Nacional del Agua; y (vii) ejecutar las disposiciones que establecidas por la Autoridad Nacional del Agua.

\subsubsection{Junta de usuarios}

Las juntas de usuarios de agua son personas jurídicas conformadas por usuarios de agua que se encuentran en el Registro Administrativo de Derechos de Uso de Agua (RADA) y previamente agrupadas en comisiones y comités de usuarios de agua para el ejercicio de su rol como operador de infraestructura hídrica (artículo 42.3 del Reglamento de la Ley de Recursos Hídricos), que se ubican en el mayor nivel de las organizaciones de usuarios de agua, sobre la base de un sector hidráulico (artículos 24 y 32 del Reglamento de la Ley de Organizaciones de Usuarios de Agua).

El reconocimiento de las juntas de usuarios comprende la autorización del Estado para brindar un servicio público, administrar, operar y mantener la infraestructura pública de un sector hidráulico, cobrar tarifas de agua y efectuar la distribución del recurso (artículo 20 del Reglamento de la Ley de Organizaciones de Usuarios de Agua).

El Reglamento de la Ley de Organizaciones de Usuarios de Agua, en el artículo 21, establece los requisitos que la junta de usuarios de agua debe cumplir para obtener el reconocimiento de la Autoridad Nacional del Agua. A continuación, se precisan los requisitos:

- Acta de constitución: acuerdo de usuarios de un mismo sector hidráulico, denominación, identificación del sector hidráulico, de los usuarios que conforman la junta de usuarios y del primer consejo directivo.

- Patrimonio de la junta constituida.

- Inventario de la infraestructura hidráulica.

- Propuesta de tarifa conforme a los lineamientos de la Autoridad Nacional del Agua.

- Propuesta de Estatuto.

- Plano o esquema del sector hidráulico, delimitado por la Autoridad Nacional del Agua.

Entre las funciones que les son reconocidas por medio del artículo 28 de la Ley de Recursos Hídricos y el artículo 25 del Reglamento de la Ley de Organizaciones de Usuarios de Agua, se encuentran las siguientes: (i) operar y mantener la infraestructura hidráulica a su cargo promoviendo su desarrollo; (ii) distribuir el agua en el sector hidráulico a su cargo, en función a la disponibilidad de los recursos hídricos y a los programas de distribución aprobados; (iii) cobrar las tarifas de agua y administrar estos recursos públicos; (iv) recaudar la retribución económica y transferir estos recursos públicos oportunamente a la Autoridad Nacional del Agua; (v) supervisar el cumplimiento de las obligaciones de los usuarios de agua del sector hidráulico; (vi) elaborar y proponer su plan de aprovechamiento de disponibilidades hídricas, incluyendo la programación de cultivo y riego de su respectivo sector hidráulico; (vii) brindar el servicio de suministro de agua en forma eficiente y atender los reclamos de los usuarios de agua del sector hidráulico dentro de los plazos previstos; participar en los Consejos de Recursos Hídricos de Cuenca, representando los intereses de los usuarios de agua del sector hidráulico a su cargo; (viii) promover y ejecutar programas y acciones de sensibilización, capacitación, difusión y asistencia técnica a favor de los usuarios de agua; elaborar estudios de preinversión, expedientes técnicos o estudios definitivos; (ix) promover la implementación de equipos, procedimientos o tecnologías que incrementen la eficiencia en el aprovechamiento sostenible de los recursos hídricos; $(\mathrm{x})$ supervisar las actividades de las comisiones de usuarios que la integran; (xi) desarrollar proyectos, o colaborar en su desarrollo, en armonía con el Plan de Gestión de Recursos Hídricos de Cuenca; (xii) promover el uso sostenible y la eficiencia en el aprovechamiento de los recursos hídricos; (xiii) promover la gestión integrada de los recursos hídricos; (xiv) representar y defender los intereses y derechos, individuales o colectivos, de los usuarios de agua del sector hidráulico a su cargo ante las entidades públicas y privadas, a nivel nacional e internacional; (xv) resolver los reclamos que formulen los usuarios de agua sobre deficiencias en la distribución del agua, o por incumplimiento de las obligaciones establecidas por ley; y (xvi) ejecutar las disposiciones que establezca la Autoridad Nacional del Agua.

La norma señala que las juntas de usuarios podrán delegar a las comisiones de 
usuarios de agua determinadas funciones a su cargo, siempre que sea por acuerdo del Consejo Directivo de aquellas y mantengan la responsabilidad por dichas funciones y servicios delegados.

Es preciso mencionar que las juntas de usuarios ejercen únicamente el rol de operador de infraestructura hidráulica menor, quedando la mayor en el ámbito del gobierno nacional y/o gobiernos regionales (artículo 26 del Reglamento de la Ley de Organizaciones de Usuarios de Agua). Por ende, las funciones previamente mencionadas se circunscriben en la infraestructura hidráulica menor.

Finalmente, se debe señalar que, aunque la normativa sobre organizaciones de usuarios de agua no lo desarrolla en su Reglamento, las comunidades campesinas y nativas son consideradas organizaciones tradicionales con los mismos derechos que las organizaciones de usuarios previamente referidas, conforme se señala en el artículo 32 de la Ley de Recursos Hídricos.

\section{Sostenibilidad de las aguas subterráneas a partir de su aprovechamiento por parte de las organizaciones de usuarios de agua}

En las secciones previas, se ha observado que, en el ordenamiento jurídico peruano, se cuenta con instrumentos normativos para la gestión de las aguas subterráneas, a partir de los cuales se establecen lineamientos para garantizar el acceso al mencionado recurso hídrico y su manejo sostenible por parte del Estado peruano. Asimismo, se ha reconocido que el régimen jurídico para el aprovechamiento de las aguas subterráneas en el país establece condiciones de sostenibilidad para el otorgamiento de derechos de uso sobre el recurso hídrico en mención. También, a partir de la normativa aplicable a los usuarios de agua con títulos habilitantes para el aprovechamiento de aguas subterráneas y a las organizaciones en las que aquellos usuarios participan, se ha reconocido la importancia de su rol en el aprovechamiento sostenible de los recursos hídricos como las aguas subterráneas, en la medida que tienen deberes y obligaciones de sostenibilidad que cumplir en el marco de sus funciones en la gestión integral de recursos hídricos.

Ahora bien, este reconocimiento de la sostenibilidad de los recursos hídricos como las aguas subterráneas que el Estado debe procurar desde los instrumentos normativos, el régimen jurídico aplicable en el otorgamiento de título habilitante para el aprovechamiento de las aguas subterráneas y las obligaciones que tienen los usuarios de agua subterránea organizados, no implica un aseguramiento material de los dichos recursos hídricos. De manera que resulta pertinente observar cómo se ha venido materializando en el aprovechamiento de las aguas subterráneas, principalmente, por parte de las organizaciones de usuarios de agua.

En principio, debemos señalar que, de acuerdo a la información brindada por la Autoridad Nacional del Agua para el 2018, en Perú, tenemos un total de 34629 pozos inventariados según tipo de uso (agrícola, doméstico, pecuario e industrial), correspondientes a 52 acuíferos distribuidos en el ámbito de competencia de las nueve Autoridades Administrativas del Agua (ANA, 2019, p. 72).

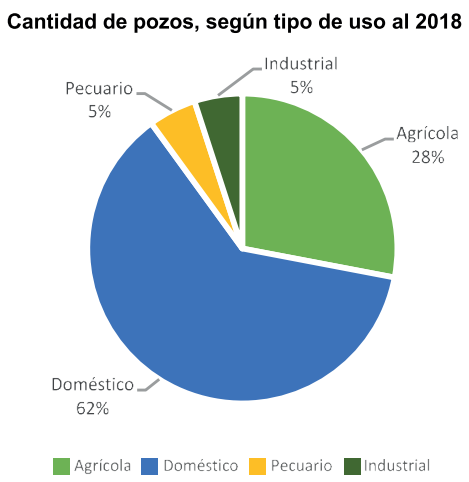

Elaboración Propia.

Fuente: ANA, 2019

El aprovechamiento de los recursos hídricos de aguas subterráneas a través de los pozos por parte de los usuarios de agua y, por ende, organizaciones de usuarios de agua, debe ser en concordancia con los instrumentos normativos sobre gestión de recursos hídricos como las aguas subterráneas, a fin de asegurar su sostenibilidad, de conformidad con lo mencionado en la segunda sección del presente artículo.

En esa misma línea, se debe reconocer que, aunque los integrantes de las organizaciones de usuarios de agua utilizan directamente las aguas subterráneas y cuentan con organizaciones propias (Rivera, 2016, p. 320 ), ello no representa una amenaza para la gestión integrada de recursos hídricos que propone el ordenamiento jurídico peruano que permita su aprovechamiento organizado y con 
criterios de sostenibilidad. De hecho, su actuación se encuentra vinculada a los instrumentos normativos de la gestión que buscan la sostenibilidad del mencionado recurso (artículo III.6 y 108 de la Ley de Recursos Hídricos), así como asegurar su aceptabilidad, disponibilidad, accesibilidad, calidad y asequibilidad como criterios que permiten definir el uso adecuado del recurso hídrico (Gronwall \& Danert, 2020, pp. 5-6).

$Y$ es que, conforme se puede apreciar de las propias funciones atribuidas a las organizaciones de usuarios de agua en la normativa aplicable, estas últimas deben cumplir determinadas funciones destinadas a la sostenibilidad y al aprovechamiento eficiente, oportuno y adecuado, tal como se puede observar en el Reglamento de la Ley de Organizaciones de Usuarios de Agua. Este deber de cumplimiento de las organizaciones de usuarios de agua se encuentra establecido en el artículo 25 del Reglamento de la Ley de Organizaciones de Usuarios de Agua para el caso de las juntas de usuarios; en el artículo 50, para las comisiones de usuarios; y, en el artículo 63 , para los comités de usuarios.

En el caso particular de las organizaciones de usuarios de aguas subterráneas, las actividades de (i) extracción, (ii) observación, (iii) medición de los usos, (iv) distribución, y (v) medición del nivel freático a realizarse en razón del aprovechamiento de dichos recursos hídricos debe ser en atención a la sostenibilidad de estos últimos. De manera que, a partir de dichas actividades, las organizaciones de usuarios de aguas subterráneas puedan y deban promover desarrollos vinculados a la recarga de acuíferos, obras de mejoramiento de infraestructura, actualización de información vinculada a los acuíferos, entre otros; bien de manera individual o a partir de coordinaciones con gobiernos regionales, gobiernos locales, autoridades locales del agua $u$ otros.

No obstante, conforme se muestra en la siguiente tabla sobre el acuífero del Valle de Ica y de las Pampas de Villacurí y Lanchas a modo de muestra, no ha sido posible observar actuaciones vinculadas a la sostenibilidad de los recursos hídricos de aguas subterráneas en el marco de las actividades que realizan los usuarios y organizaciones de usuarios de este recurso hídrico, sino que, por el contrario, tienden a la sobreexplotación del recurso hídrico en mención (Zeisser \& Gilvonio, 2016, pp. 6-7).

\section{Reservas explotables y volúmenes otorgados por} licencia en hectómetros cúbicos $\left(\mathrm{hm}^{3}\right)$

\begin{tabular}{|l|c|c|c|c|c|}
\hline Acuífero & $\begin{array}{c}\text { Reserva } \\
\text { explotable }\end{array}$ & Explotación & $\begin{array}{c}\text { Sobreexplo- } \\
\text { tación }\end{array}$ & $\begin{array}{c}\text { Volumen } \\
\text { otorgado }\end{array}$ & $\begin{array}{c}\text { Volumen asig- } \\
\text { nable (restante) }\end{array}$ \\
\hline Ica & 189 & 335 & 146 & 134.14 & 54.86 \\
\hline Villacurrí & 63 & 228 & 165 & 87.8 & -24.8 \\
\hline Lanchas & 17 & 34 & 17 & 3.5 & 13.5 \\
\hline
\end{tabular}

Elaboración propia.

Fuente: Plan de gestión del acuifero del Valle de Ica y Pampas de Villacuri y Lanchas, citado por Zeisser y Gilvonio, 2016.
En la tabla presentada, podemos observar que, aun cuando existente un máximo de reserva explotable de aguas subterráneas, se ha llegado a sobrexplotar dichos recursos en los acuíferos de Ica, Villacurrí y Lanchas en 146, 165 y 17 hectómetros cúbicos $\left(\mathrm{hm}^{3}\right)$, respectivamente. Se debe destacar que, aun cuando la sobreexplotación del recurso hídrico en mención no sea alegada íntegramente a los usuarios de agua organizados con licencias de agua otorgadas por la autoridad competente, es posible observar que hay usuarios de agua subterránea con licencias de uso que llegan a explotar el recurso hídrico más allá de lo permitido por la reserva explotable, como es el caso del acuífero de Villacurrí, en donde el volumen otorgado es $24.8 \mathrm{hm} 3$ superior de lo establecido.

En atención a dicha situación de sobreexplotación del recurso hídrico mencionado, merece especial referencia conocer la situación de los derechos de uso de agua subterránea en Ica que le fueron reconocidos a través de títulos habilitantes de licencia de uso. Para el año 2014, según información de la Administración Local del Agua Ica en 2015, citada por Zeisser y Gilvonio, se otorgaron 270 licencias de uso de agua subterránea para actividades agrarias con un volumen de $146.4 \mathrm{hm} 3,43$ licencias de uso de agua para el poblacional con un volumen de $14 \mathrm{hm} 3$ y 6 licencias de uso de agua para otros usos como el doméstico agropecuario, pecuario, recreativo e industrial. (2016, p. 9). De ahí que, según anotan los mismos autores, del registro de licencias de uso de agua subterránea de la Administración Local del Agua Ica en 2014, de acuerdo a la información recogida por esta última en el 2015 para la cobranza de retribución económica respectiva, se observa la grave afectación del acuífero de Ica, en tanto la sobreexplotación estaba cuantificada en 146 hm3 anualmente. (2016, p. 11).

De manera que el aprovechamiento sostenible de las aguas subterráneas otorgadas a los usuarios de agua mediante título habilitante todavía sea una afirmación que se mantiene en el plano normativo, a partir del cual Estado deberá adoptar las acciones que correspondan a efectos de asegurar la sostenibilidad del recurso hídrico 
en mención. Sin perjuicio de que, como ya se esbozado en líneas anteriores, los usuarios de agua también coadyuvan en la sostenibilidad de los recursos en el marco de los derechos de uso de agua que les fueron otorgados y, además, aportan desde sus ámbitos de organización en calidad de juntas, comisiones o comités de usuarios de aguas en atención a los deberes y obligaciones derivadas de sus funciones en la gestión integral de recursos hídricos.

En ese sentido, aun cuando exista una debilitada comprensión del deber de coadyuvar a la sostenibilidad de los recursos hídricos, como las aguas subterráneas, por parte de los usuarios de agua a consecuencia de una cohesión social debilitada que olvide la afectación que genera la sobreexplotación de dichos recursos sobre terceros, las organizaciones de usuarios de agua tienen del deber y la obligación de observar que las actuaciones de los usuarios de agua que los integran cumplan y/o se limiten al volumen de aguas subterráneas que les fue permitido aprovechar para los diferentes usos reconocidos por la norma correspondiente.

Sin perjuicio de que los usuarios de agua organizados en juntas, comisiones o comités realicen las acciones para alcanzar la sostenibilidad de las aguas subterráneas, el Estado ha venido implementando herramientas institucionales para garantizar el uso sostenible de los mencionados recursos hídricos. Así, una de ellas es la Guía para realizar inventarios de fuentes de aguas subterráneas, emitida por Resolución Jefatural 086-2020-ANA de la Autoridad Nacional del Agua.

Otra de las herramientas es el Directorio de las Organizaciones de Usuarios de Agua de la Autoridad Nacional del Agua, el cual, sin embargo, debería seguir trabajándose en aras de aumentar las organizaciones de usuarios de agua subterránea en particular (2020) y superar las dificultades que puedan presentarse para su creación como los problemas de representatividad legal, problemas para administrar los fondos para la operación de la infraestructura hidráulica, los problemas con los pagos oportunos de las tarifas y la falta de elaboración de planes para la operación de la infraestructura hidráulica, según lo anotado por la Autoridad Nacional del Agua en su participación como amicus curiae en el proceso de los Expedientes 0018-2014-PI/TC y 0022-2014-PI/TC, acumulados.

En esa misma línea, otra de las herramientas institucionales es que la Autoridad Nacional del Agua se encuentra habilitada para declarar zonas de veda (o también zonas de restricción, en caso de que así corresponda) en atención a la gestión sostenible de los recursos hídricos como las aguas subterráneas (numeral 6 del artículo 15, 78 y 113 de la Ley de Recursos Hídricos) y, con ello, los usuarios de agua tengan restringido su aprovechamiento durante el tiempo y en el lugar sobre el que se haya declarado zona de veda o zona de protección. A modo de ejemplo, mediante Resolución Jefatural
330-2011-ANA, la Autoridad Nacional del Agua había ratificado la condición de veda de los acuíferos de Ica, Villacurí y Lanchas, debido a la sobreexplotación de aguas subterráneas en estos acuíferos, situación que ha persistido hasta hace unos años como se ha advertido en líneas anteriores.

En razón de lo expuesto en este apartado, podemos señalar que el Estado peruano tiene el deber de garantizar el uso sostenible de los recursos hídricos como las aguas subterráneas, pero que, a su vez, los usuarios de agua organizados también tienen el deber y la obligación de coadyuvar en la sostenibilidad de dichos recursos a fin de evitar situaciones de sobreexplotación de los mencionados recursos hídricos. En aras de que las organizaciones de usuarios de agua puedan generar un impacto sobre la sostenibilidad de las aguas subterráneas, será necesario el fortalecimiento de dichas organizaciones para que se pueda aprovechar los beneficios que se generarían por un buen funcionamiento de las mismas para la gestión integrada de recursos hídricos en el país.

\section{Conclusiones}

A partir de lo expresado en las secciones de desarrollo, podemos arribar a algunas conclusiones sobre el rol de las organizaciones de usuarios de agua en el aprovechamiento sostenible de las aguas subterráneas en el país. Así, en primer lugar, debemos comenzar por destacar la importancia del agua subterránea para el bienestar del ser humano, al ser primordial en el suministro de agua para el ámbito urbano y rural, conforme anotan Foster et al. (2002, p. 2) y Villar (2016, pp. 93-96).

En segundo lugar, en aras de dicha importancia, el Estado peruano tiene el deber y obligación de garantizar el uso sostenible de los recursos hídricos, en los que están incluidas las aguas subterráneas. De ahí que establezca instrumentos normativos para asegurar la gestión sostenible de dichos recursos $y$, a su vez, el régimen jurídico aplicable con condiciones de sostenibilidad para el otorgamiento de derechos de usos a usuarios de agua para su aprovechamiento. 
En tercer lugar, debemos reconocer el rol que cumplen las organizaciones de usuarios de agua en el aprovechamiento de las aguas subterráneas que realizan sus miembros, quienes son usuarios de agua con títulos habilitantes. $Y$ es que, sea una junta, una comisión o un comité, las organizaciones de usuarios de agua tienen el deber y la obligación de coadyuvar a la sostenibilidad del aprovechamiento de los recursos hídricos por parte de los usuarios de agua, a partir de las funciones atribuidas por la normativa aplicable a dichas organizaciones.

En cuarto lugar, será preciso mencionar que, aun cuando el Estado tenga el deber primario de garantizar la sostenibilidad en el uso de los recursos hídricos como las aguas subterráneas, las organizaciones de usuarios de agua cumplen un rol relevante en dicha sostenibilidad, a partir del aprovechamiento de los recursos hídricos de parte de sus miembros y desde el deber que se les ha atribuido por su participación en la gestión integral de los recursos hídricos. No obstante, conforme a lo anotado en líneas anteriores, todavía hay escenarios de sobreexplotación de las aguas subterráneas que generan efectos negativos para la sostenibilidad de dichos recursos hídricos, frente a los cuales se deberá buscar adoptar medidas para la conservación de los recursos hídricos que aprovechan a partir de una diligente extracción, observación, medición del uso, distribución y medición del nivel freático, sin perjuicio de las herramientas institucionales que ha venido implementando el Estado para garantizar el uso sostenible de las aguas subterráneas.

Finalmente, resulta pertinente reconocer que, aun cuando se haya mencionado la importancia del rol, normativa aplicable y deber de las organizaciones de usuarios de agua en el aprovechamiento sostenible de las aguas subterráneas en Perú, todavía resulta una tarea pendiente la implementación de herramientas institucionales que permitan asegurar la sostenibilidad desde las organizaciones de usuarios de agua en relación al recurso hídrico en mención, por parte de las autoridades competentes.

\section{Referencias bibliográficas}

Autoridad Nacional del Agua, Banco Interamericano de Desarrollo \& Banco Mundial. (s.f.). Plan de Gestión de Recursos Hídricos de Cuenca. ANA, Banco Interamericano de Desarrollo y Banco Mundial.

Autoridad Nacional del Agua. (2009, 11 de mayo). Resolución Jefatural 0250-2009-ANA. Política y Estrategia Nacional de Recursos Hídricos.

Autoridad Nacional del Agua (2011, 08 de junio). Resolución Jefatural 330-2011-ANA.

Autoridad Nacional del Agua. (2015, 10 de diciembre). Resolución Directoral N 006-2015-ANA-DCPRH. Lineamientos para la aprobación de Planes de Gestión de Recursos Hídricos en las Cuencas.

Autoridad Nacional del Agua (2016). Planificación Hídrica en el Perú. ANA. https://www.ana.gob.pe/sites/default/files/publication/files/ planificacion_hidrica_en_el_peru.pdf
Autoridad Nacional del Agua. (2018, 29 de octubre). Resolución Jefatural 327-2018-ANA. Reglamento de Operadores de Infraestructura Hidráulica.

Autoridad Nacional del Agua. (2019). Compendio Nacional de Estadísticas de Recursos Hídricos. ANA y Sistema Nacional de Información de Recursos Hídricos.

Autoridad Nacional del Agua. (2020, 22 de mayo). Resolución Jefatural 086-2020-ANA. Guía para realizar inventarios de fuentes de agua subterránea.

Autoridad Nacional del Agua. (2020). Directorio de las Organizaciones de Usuarios de Agua [base de datos]. ANA. https://www.ana.gob.pe/ organizaciones-de-usuarios-/directorio-de-lasorganizaciones-de-usuarios-de-agua

Cairampoma, A. \& Villegas, P. (2016). Régimen jurídico de las aguas subterráneas en el Perú, Themis-Revista de Derecho, 69, 147-158.

Congreso de la República. (2014, 19 de enero). Ley 30157. Ley de Organizaciones de Usuarios de Agua.

Congreso de la República. (2009, 30 de marzo). Ley 29338. Ley de Recursos Hídricos.

Foster, S., Hirata, R., Gomes, D., D’Elía, M. \&, París, M. (2002). Protección de la Calidad del Agua Subterránea: guía para empresas de agua, autoridades municipales y agencias ambientales. Banco Mundial

García de Enterría, E. (1984). Revolución francesa y Administración contemporánea. Taurus.

Garduño, H., Foster, S., Dumars, C., Kemper, K., Tuinhof, A. \&, Nanni, M. (2006). Derechos de Extracción de Agua Subterránea, de la teoría a la práctica. En Gestión del agua subterránea: conceptos y herramientas. Banco Mundial.

Gronwall, J. \& Kerstin, D. (2020). Regarding groundwater and drinking water access through a human rights lens: self-supply as a norm. Water, 12(2), 419. https://doi.org/10.3390/w12020419

Groundwater Governance. (2016). Global diagnostic on Groundwater Governance. Food and Agriculture Organization of the United Nations.

Izquierdo, M. (2019). Fiscalización, supervisión e inspección administrativa: aproximación conceptual crítica y caracteres generales en el Derecho Peruano. En La Proyección del Derecho Administrativo Peruano. Estudios por el Centenario de la Facultad de Derecho de la PUCP. Palestra Editores.

López-Vera, F. (2012). Groundwater: the invisible resource. International Journal of Water Resources Development, 28(1), 141-150. 
Lozano, B. (2015). Modificaciones y vicisitudes de la planificación hidrológica. Fundación Agbar y Consejo General del Poder Judicial.

Martín Mateo, R. (1991). Administración de los recursos hídricos. Aspectos institucionales y modalidades gestoras. Revista de Administración Pública, 124, 7-34.

Muñoz, D. (2016). Agro-exportación y sobreexplotación del acuífero de Ica en Perú. Anthropologica, 34(37), 115-138.

Parejo Alfonso, L. (2012). Lecciones de Derecho Administrativo. Tirant lo Blanch.

Pino, E. (2019). El acuífero costero La Yarada, después de 100 años de explotación como sustento de una agricultura en zonas áridas: una revisión histórica. Idesia, 37(3), 39-45.

Presidencia de la República. (2009, 22 de mayo). Decreto Supremo 12-2009-MINAM. Política Nacional Ambiental.

Presidencia de la República. (2010, 23 de marzo). Decreto Supremo 001-2010-AG. Reglamento de la Ley de Recursos Hídricos.

Presidencia de la República. (2015, 03 de abril). Decreto Supremo 0005-2015-MINAGRI.
Reglamento de la Ley de Organizaciones de Usuarios de Agua.

Presidencia de la República. (2015, 15 de julio). Decreto Supremo 013-2015-MINAGRI. Plan Nacional de los Recursos Hídricos.

Presidencia de la República. (2019, 25 de enero). Decreto Supremo 004-2019-JUS. Texto Único Ordenado de la Ley de Procedimiento Administrativo General.

Rivera, D. (2016). Gestión colectiva y conjunta de aguas: perspectiva jurídica de una deuda subterránea. Revista de Derecho de la Pontificia Universidad Católica De Valparaíso, 46, 311-346.

Sánchez Morón, M. (2011). Derecho Administrativo. Parte General. Tecnos.

SUNARP. (2018). Guía General para la inscripción de actos de las organizaciones de usuarios de agua. SUNARP.

Tribunal Constitucional (2020). Sentencia recaída en los Expedientes 0018-2014-PI/TC y 0022-2014-PI/TC Acumulados.

Tribunal Constitucional (2020, 14 de enero). Expediente 0006-2019CC/TC (Espinosa-Saldaña Barrera, E.).

Villar, P. (2016). As águas subterrâneas e o direito à água em um contexto de crise. Ambiente \& Sociedade, 19(1), 83-102.

Zeisser, M. \& Gilvonio, A. (2016). Estudio de caso: Plan de Gestión del Acuífero de lca y el Proceso de Formalización-Regularización de Pozos. CEPES, CODEHICA, SER, SISAY y COOPERACCIÓN. 
